\title{
Effects of inhomogeneous myofibril morphology on optical diffraction in single muscle fibers
}

\author{
Janaka Ranasinghesagara and Gang Yao* \\ Department of Biological Engineering, University of Missouri-Columbia, Columbia, Missouri 65211, USA \\ *Corresponding author: yaog@missouri.edu
}

Received May 13, 2008; revised October 11, 2008; accepted October 15, 2008; posted October 16, 2008 (Doc. ID 96035); published November 21, 2008

\begin{abstract}
Laser diffraction is commonly used in physiological research that explores single muscle fibers. Although variations in sarcomere morphological properties have often been observed, their effects on laser diffraction have not been studied in detail. In this study, we applied three-dimensional coupled wave theory to a physical sarcomere model to investigate the effects of inhomogeneous morphological profiles in muscle fibers. The simulation results were compared with several those of published experimental studies. Our results indicate that by incorporating various myofibril inhomogeneities such as skew and domain effect in the theoretical model, a variety of observations in single fiber diffraction under different experimental conditions can be reproduced in the simulation. (C) 2008 Optical Society of America

OCIS codes: $050.1960,170.6935,170.3660,260.1960$.
\end{abstract}

\section{INTRODUCTION}

Skeletal muscles contribute about $40 \%$ of human body weight and are important for many physiological functions. Whole muscle consists of many fascicles, or collections of muscle fibers (muscle cells), encapsulated in an elaborate connective tissue matrix. There are hundreds of small myofibrils within each single muscle fiber. The contraction of skeletal muscles is responsible for force generation and body movement. Muscle contraction is realized by the fundamental repetitive unit in myofibrils, the sarcomere [1], via the interactions of the actin and myosin proteins during cross-bridge cycling. Under an optical microscope, a sarcomere appears as alternating light and dark bands, termed I-bands and A-bands, respectively. Because of such obvious striations, light diffraction has been widely applied to study sarcomere dynamics in single muscle fibers [2-5].

Classical diffraction theories have been applied to study the diffraction of light by muscle fibers [6-9]. Although they provide good explanations for the relationship between sarcomere length and diffraction angles, most of these early theories fail to explain the effects of fiber thicknesses on diffraction efficiency. Huxley [10] was the first to model the sarcomere as a thick grating unit. He considered wave coupling and studied how constructive and destructive interference can change diffraction intensity across the fiber. However, his model was not capable of analyzing muscle fibers with different striation patterns. On the basis of the work of Huxley and Hanson's work [11] on refractive index modulation in sarcomeres, Thornhill et al. [12] described a much improved sarcomere model and applied the first-order coupled wave theory developed by Kogelnik [13]. Sidick et al. [14] further introduced rigorous coupled wave analysis for planar diffraction (2DCW) [15] to analyze light diffraction in muscle fibers for TE polarization. Their method was later extended to study the muscle birefringence effect [16].
Despite multiple previous attempts to develop an accurate model to describe sarcomere diffraction, few studies have attempted to explain the many inconsistencies observed in the experimental results. For example, the efficiency of first-order diffraction was shown to increase with sarcomere length by Baskin et al. [3]. Others [2] have reported a curvilinear relationship. We hypothesized that such apparent inconsistencies can be explained if the inhomogeneous nature of the muscle fibers was taken into consideration. Most of the previous theoretical studies assumed that muscle fiber had a uniform and wellorganized myofibril arrangement featuring straight, skewed, or sinusoidal ripple striations $[7,12,17-19]$. On the other hand, microscopic studies have shown that morphological and optical parameters do not remain constant throughout a myofibril [20-23].

In general, a muscle fiber can be divided into small domains with relatively uniform myofibril properties. When illuminated by a wider light beam, the resulting diffraction is the sum of the various levels of diffraction from all the contributing domains [21,22]. Different domains may have different morphological parameters. Sidick et al. [16] found that a random skew effect should be included to explain their birefringence experimental results. However, such inhomogeneous morphology effects have not been studied in detail using a theoretical framework.

In this study, we developed an improved sarcomere diffraction model by considering various myofibril inhomogeneities and the domain effect. The new model is consistent with other published data. We applied threedimensional coupled wave analysis for conical diffraction (3DCW) [24] to simulate the diffraction of light by muscle fibers. In contrast to the coupled wave analysis for planar diffraction (2DCW) [15] that was used in other studies, $3 \mathrm{DCW}$ is appropriate for any incidence angle on any incidence plane with either TE or TM polarization. Accordingly, $3 \mathrm{DCW}$ is more flexible and is a promising tool for 
analyses of light propagation in whole muscle [25]. The simulation results were quantitatively compared with previously reported experimental results on single muscle fibers. We found that a variety of experimental observations can be explained more accurately by the new model that considers inhomogeneous sarcomere morphologies.

\section{METHODS}

\section{A. Three-Dimensional Rigorous Coupled Wave Analysis} for Conical Diffraction (3DCW)

Three-dimensional rigorous coupled wave analysis for conical diffraction was formulated by Moharam and Gaylord [24] to analyze planar and surface relief gratings mounted at an angle. Since then, it has been widely used to analyze diffractive structures [26,27]. Following their derivation [24], the incidence region is labeled I and the transmitted region is defined as III. As shown in Fig. 1, $\mathbf{k}_{\mathrm{I}}$ is the vector of the wave that is obliquely incident on the grating at an incidence angle of $\boldsymbol{\alpha}$ and an azimuthal angle of $\boldsymbol{\delta}$. The electrical fields in regions I and III can be expressed as

$$
\begin{aligned}
\mathbf{E}_{\mathrm{I}} & =\mathbf{E}_{0} \exp \left(-j \mathbf{k}_{\mathrm{I}} \cdot \mathbf{r}\right)+\sum_{i} \mathbf{R}_{i} \exp \left[-j \mathbf{k}_{\mathrm{I} i} \cdot \mathbf{r}\right], \\
\mathbf{E}_{\mathrm{III}} & =\sum_{i} \mathbf{T}_{i} \exp \left[-j \mathbf{k}_{\mathrm{III} i} \cdot(\mathbf{r}-\mathbf{d})\right],
\end{aligned}
$$

where $\mathbf{E}_{0}$ is the incident electrical field; $\mathbf{d}$ is the fiber thickness; $\mathbf{R}_{i}$ and $\mathbf{T}_{i}$ are the electrical fields of the $i$ th reflected and transmitted waves, respectively; and $\mathbf{k}_{\mathrm{I} i}$ and $\mathbf{k}_{\mathrm{III} i}$ are the wave vectors in regions I and III, respectively.

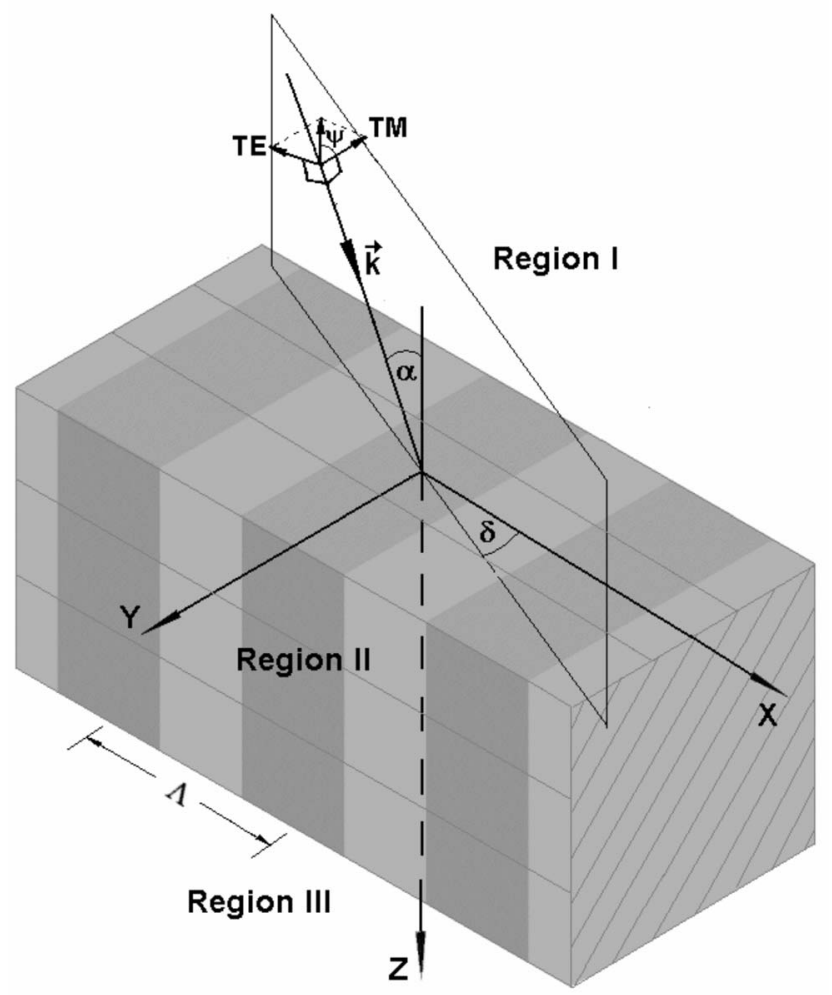

Fig. 1. Coordinates used in the 3DCW simulation of a sarcomeric grating structure.
In region II, the transverse electrical field $\mathbf{E}_{\mathrm{II}}$ and the magnetic field $\mathbf{H}_{\mathrm{II}}$ can be expressed in terms of the space harmonics $\mathbf{S}$ and $\mathbf{U}$ as

$$
\begin{aligned}
\mathbf{E}_{\mathrm{II}}= & \sum_{i}\left[S_{x i}(z) \hat{x}+S_{y i}(z) \hat{y}+S_{z i}(z) \hat{z}\right] \exp \left[-j \boldsymbol{\sigma}_{i} \cdot \mathbf{r}\right], \\
\mathbf{H}_{\mathrm{II}}= & \sqrt{\epsilon_{0} / \mu_{0}} \sum_{i}\left[U_{x i}(z) \hat{x}+U_{y i}(z) \hat{y}+U_{z i}(z) \hat{z}\right] \\
& \times \exp \left[-j \boldsymbol{\sigma}_{i} \cdot \mathbf{r}\right],
\end{aligned}
$$

where $\epsilon_{0}$ and $\mu_{0}$ are the free space permittivity and permeability, respectively, and $\sigma_{i}=k_{x i} \hat{x}+k_{y} \hat{y}$ when the grating is perpendicular to the $z$ axis [28]. The set of coupled wave equations of $\mathbf{S}$ and $\mathbf{U}$ can be derived by substituting Eqs. (3) and (4) into Maxwell's equations:

$$
\begin{aligned}
& \nabla \times \mathbf{E}_{\mathrm{II}}=-j \omega \mu_{0} \mathbf{H}_{\mathrm{II}}, \\
& \nabla \times \mathbf{H}_{\mathrm{II}}=j \omega \epsilon_{0} \epsilon(x) \mathbf{E}_{\mathrm{II}},
\end{aligned}
$$

where $\epsilon(x)$ is the permittivity distribution in the grating, and is the square of the refractive index profile: $\epsilon(x)$ $=n^{2}(x)$. The distribution of permittivity in the grating $\epsilon(x)$ is independent of $\hat{z}$ because the grating is perpendicular to the $z$ axis. The Fourier expansion of $\epsilon(x)$ can be written as

$$
\epsilon(x)=\sum_{h} \hat{\epsilon}_{h} \exp (j h \mathbf{K} x)
$$

where $\mathbf{K}$ is the grating vector $\mathbf{K}=2 \pi / \Lambda$, and $\Lambda$ is the grating period. The $h$ th Fourier coefficient is given by

$$
\hat{\epsilon}_{h}=\frac{1}{\Lambda} \int_{0}^{\Lambda} \epsilon(x) \exp (-j h \mathbf{K} x) \mathrm{d} x .
$$

The derived coupled wave equation can be solved by applying the Floquet condition and continuous boundary conditions. Stable and efficient numerical algorithms for 3DCW have been previously developed by different groups [28-30]. The final diffraction efficiencies of each diffraction order for transmitted light (in region III) can be calculated as

$$
\mathrm{DE}_{\mathrm{III} i}=\operatorname{Re}\left(k_{z \mathrm{III} i} / k_{z}\right)\left|\mathbf{T}_{i}\right|^{2},
$$

where $\mathrm{DE}_{\mathrm{III} i}$ is the $i$ th-order diffraction efficiency in region III; $k_{z}$ is the $z$ component of $\mathbf{k}_{\mathrm{I}}\left(k_{z}=k_{\mathrm{I}} \cos \alpha\right)$; and $k_{z \mathrm{III} i}$ is the $z$ component of the $i$ th diffracted wave vector in region III. 3DCW can be used for either TE or TM polarized light; calculations from both polarizations can subsequently be averaged to predict results for unpolarized incident light.

\section{B. Sarcomere Model and Parameters}

A sarcomere has a well-organized structure with a periodic distribution of refractive indices based on the relative concentrations of two major proteins known as actin and myosin [1]. A sarcomere's striated appearance is mainly due to the alternating darker A-bands and lighter I-bands [Fig. 2(a)]. The segment of the A-band with only myosin proteins is called the $\mathrm{H}$ zone. The Z-line and M-line are located in the middle of the I-band and in the middle of the $\mathrm{H}$ zone, respectively. The sarcomere length is usually 


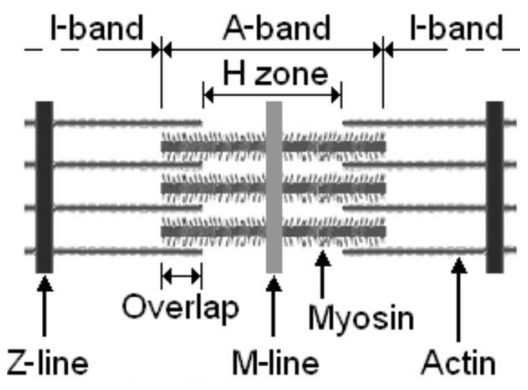

(a)

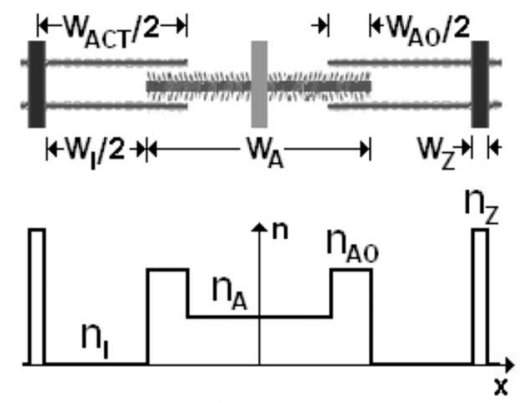

(b)

Fig. 2. Illustrations of (a) the sarcomere structure and (b) the optical sarcomere model. The distribution of refractive indices is also shown. $W_{A C T}, W_{A}, W_{I}, W_{A O}$, and $W_{Z}$ are the widths of the actin filament, A-band, I-band, overlap region, and Z-line, respectively. $n_{A}, n_{I}$, $n_{A O}$, and $n_{Z}$ are the refractive indices of the A-band, I-band, overlap region, and Z-band, respectively.

defined as the distance between two Z-lines. The overlap region refers to the part of the A-band where both actin and myosin are present.

The sarcomere model proposed by Thornhill et al. [12] was used in this study. This model improved the simpler Huxley model [10] by considering seven subregions in a single sarcomere [Fig. 2(b)]. These included the overlap region, the $\mathrm{H}$ zone, and the $\mathrm{Z}$-line. We included the actual Z-line thickness in our study when calculating the Fourier coefficients of the refractive index distribution. We calculated the exact Fourier components [Eq. (8)] of these structures. Our calculations indicated that inclusion of the M-line has limited effect $(<11 \%$ on diffraction efficiencies) on our results.

The majority of the reported experiments involving laser diffraction were conducted in frog semitendinosus muscles or rabbit psoas muscles. In most cases, the sarcomere length $\Lambda$ varied between 2.0 and $4.0 \mu \mathrm{m}$ [2,9]. In our simulation, all sarcomere parameters were chosen based on experimental measurements. From published data [31-38], the typical physiological ranges of several major sarcomere parameters are shown in Table 1. Unless specified individually, the following default parameters

Table 1. Muscle Sarcomere Parameters of the Frog Semitendinosus and Rabbit Psoas Muscles Reported in Published Studies

\begin{tabular}{ccccc}
\hline Parameter & Species & Muscle & Size $(\mu \mathrm{m})$ & Reference \\
\hline \multirow{4}{*}{$W_{A}$} & Frog & Semitendinosus & $1.2-1.6$ & {$[31]$} \\
& Frog & Semitendinosus & $1.47-1.59$ & {$[32]$} \\
& Frog & Semitendinosus & $1.43-1.64$ & {$[33]$} \\
& Rabbit & Psoas & 1.63 & {$[34]$} \\
& & & & \\
$W_{A C T}$ & Frog & Semitedinosus & $1.87-1.97$ & {$[32]$} \\
& Frog & Semitedinosus & $1.70-1.95$ & {$[33]$} \\
& Rabbit & Psoas & 2.24 & {$[34]$} \\
& & & & \\
$W_{Z}$ & Frog & Semitendinosus & $0.035-0.054$ & {$[35]$} \\
& Frog & Semitendinosus & $\approx 0.08$ & {$[36]$} \\
& & & & \\
& Frog & Semitendinosus & $35-105$ & {$[23]$} \\
& Frog & Semitendinosus & $38-103$ & {$[37]$} \\
& Rabbit & Psoas & $60-100$ & {$[38]$} \\
\hline
\end{tabular}

were used in our model. The width of the A-band or thick filament $\left(W_{A}\right)$ was $1.5 \mu \mathrm{m}$. The widths of the thin filament $\left(W_{A C T}\right)$ were 1.9 and $2.0 \mu \mathrm{m}$ for frog and rabbit muscle, respectively. The width of the Z-line $\left(W_{Z}\right)$ was $0.05 \mu \mathrm{m}$ for all muscles. The length of the overlap area of the A-band $\left(W_{A O}\right)$ can be calculated as $W_{A O}=W_{A}+W_{A C T}$ $+W_{Z}-\Lambda$. The I-band length can be calculated as $W_{I}$ $=W_{A C T}-W_{A O}$. The myofibril thickness and the fiber diameter (D) were $1.0 \mu \mathrm{m}$ and $80 \mu \mathrm{m}$, respectively, following the approach used in previous simulation studies $[12,14,16]$.

The wavelength $\lambda$ of the incident light was $633 \mathrm{~nm}$. The refractive indices of each sarcomere unit were largely based on the results obtained by Huxley and Hanson [11] and by Thornhill et al. [12]. The refractive index mismatch between the I-band and the overlap A-band was $\Delta n=0.0074$. The refractive index of the I-band was $n_{I}$ $=1.3800$, and the refractive index of the overlap area in the A-band was calculated as $n_{A O}=n_{I}+\Delta n=1.3874$. The refractive index of the $\mathrm{H}$ zone was $n_{H}=n_{I}+\Delta n / 2=1.3837$ [12]. The refractive index of the Z-line was estimated as $n_{Z}=0.11\left(n_{A O}-n_{I}\right)\left(W_{A} / W_{Z}\right)[11]$.

It should be noted that the refractive index mismatch $\Delta n$ varies with the sarcomere length [16] consistent with experimental observations [31]. Similar to a previous study [39], we assumed that $\Delta n$ was proportional to the square of the sarcomere length. We selected an appropriate proportionality constant by equating a $\Delta n$ of 0.0074 to a sarcomere length of $3.04 \mu \mathrm{m}$. When the fiber is overstretched, the A-band and the I-band can be completely separated and the overlap region disappears. The refractive index of this region was assumed to be the mean refractive index of the sarcomere.

\section{Morphological Variations in Myofibril Organization} In a completely straight skeletal muscle fiber [Fig. 3(a)], sarcomeres are perfectly aligned so that the Z-lines form a straight line that is perpendicular to the muscle surface. However, actual muscle fibers may possess one or more morphological variations such as skew, ripple, or slip as observed in electron micrographs [22]. As shown in Figs. $3(\mathrm{~b})-3(\mathrm{e})$, these structural variations can be modeled in terms of misalignments of the Z-line among myofibrils. In other words, the phase of the Fourier coefficient in Eq. (8) 


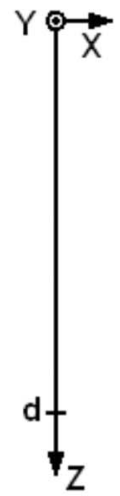

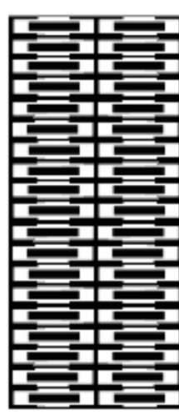

(a)

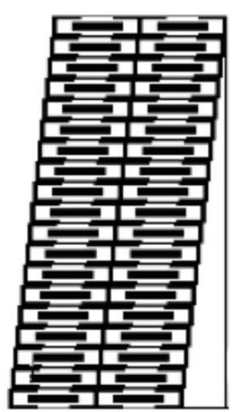

(b)

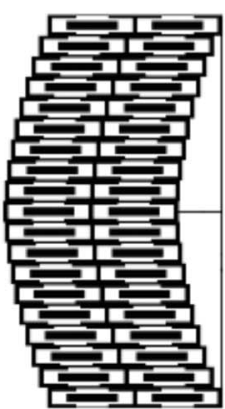

(c)

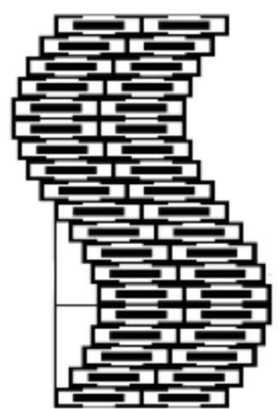

(d)

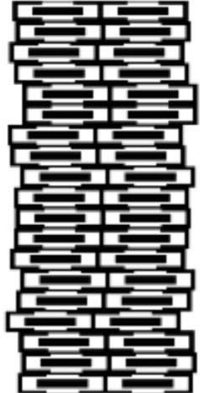

(e)

Fig. 3. Schematic illustration of muscle fibers of different morphologies. (a) Straight, (b) skew, (c) half-wave ripple, (d) full-wave ripple, (e) random slip.

is shifted by $u(z)$ for a specific myofibril located at $z$, where $u(z)$ is the distance between the center of a sarcomere and the $z$ axis $[12,14]$.

In skewed muscle fibers [Fig. 3(b)], the displacement function $u(z)$ is a linear function of the depth $z$ :

$$
u(z)=z \tan \alpha_{\text {skew }},
$$

where $\alpha_{\text {skew }}$ is the slant angle. The $u(z)$ is positive or negative depending on whether the skew is toward the left or toward the right with respect to the $z$ axis. For curved striations [ripple, Figs. 3(c) and 3(d), the $u(z)$ can be represented as a sinusoidal striation [12]:

$$
u(z)=u_{\text {ripple }} \sin \left(2 \pi z / \lambda_{\text {ripple }}\right),
$$

where the ripple amplitude $u_{\text {ripple }}$ indicates the maximum displacement distance. $\lambda_{\text {ripple }}$ is the periodic distance of the ripple. As in previous studies, two special cases can be considered: the half-wave ripple $\left[\lambda_{\text {ripple }}=2 d\right.$, Fig. $\left.3(\mathrm{c})\right]$ and the full-wave ripple $\left[\lambda_{\text {ripple }}=d\right.$, Fig. $\left.3(\mathrm{~d})\right]$, where $d$ is the fiber thickness. The slip of a myofibril [Fig. 3(e)] is a phenomenon in which $u(z)$ becomes a random variable [16] For a normal random distribution,

$$
u(z)=N\left(0, \delta_{\text {slip }}^{2}\right),
$$

where $N\left(0, \delta_{\text {slip }}^{2}\right)$ is a normally distributed random variable with a zero mean and $\delta_{\text {slip }}^{2}$ variance. For convenience, $\delta_{\text {slip }}$ is represented as a percentage of the sarcomere length $\Lambda$ in Section 3.

The misalignment of the Z-line in myofibrils can be modeled theoretically by modifying the Fourier coefficients for each myofibril. The 3DCW was applied to calculate the diffracted electric and magnetic fields in each myofibril. The fields generated by the previous myofibril acted as incident fields for the subsequent myofibril. The process was repeated until light was diffracted from the last myofibril.

D. Domain Effect and Variable Sarcomere Parameters At different locations along a muscle fiber, the structural profiles of the myofibril might be different. It is reasonable to assume that a specific set of morphological parameters can be used for a small area of the muscle fiber (a small domain), while different parameters may be assigned to different areas. The domain effect can be af- fected by many sarcomere parameters, including skew, ripple, slip, sarcomere length, etc. However, in practice skew is the variable most often encountered in real samples, and it has a significant impact on diffraction efficiency. In almost all the reported experimental studies, at least a small shift from the theoretical Bragg peak location was observed. Bragg incident angle $\theta$ is defined by $\sin \theta=m \lambda / 2 \Lambda$, where $m$ is the diffraction order. These observations suggest that skew is indeed a prevalent morphology that exists in muscle fibers. Electron microscopic studies [22] have shown that the skew angles can be as large as $20^{\circ}$. Accordingly, in this study we focused primarily on the skew-induced domain effect. The skew domain effect can be simulated by assigning a random slant angle $\alpha_{i}$ to each domain along a single myofibril:

$$
\alpha_{i}=N\left(\alpha_{\mu}, \alpha_{\nu}^{2}\right)
$$

where $N\left(\alpha_{\mu}, \alpha_{v}^{2}\right)$ is a normally distributed random variable with a mean slant angle $\alpha_{\mu}$ and a variance of $\alpha_{v}^{2}$. The diffracted electrical and magnetic fields [Eqs. (1)-(6)] from each myofibril were calculated by integrating contributions from every individual domain. These total fields were then applied as incident fields to the next myofibril. The process was repeated until the light passing through the entire muscle fiber and the final diffraction efficiency could be calculated using Eq. (9).

\section{RESULTS AND DISCUSSION}

Most previous experimental studies that explored light diffraction in single muscle fibers can be classified into one of two categories. The first category is related to the Bragg diffraction phenomenon $[5,12,21,23,40]$. In such studies, diffraction efficiencies were measured as a function of incidence angles ( $\omega$ scan). In the second category, light diffraction efficiencies were measured at different sarcomere lengths $[2,3,9,41]$ by stretching the muscle fibers.

\section{A. Diffraction Efficiencies as a Function of Incidence Angle ( $\omega$ Scan)}

When incident light satisfies the Bragg condition, the diffracted light reaches the maximum or minimum diffraction efficiency depending on the thickness of the volumet- 

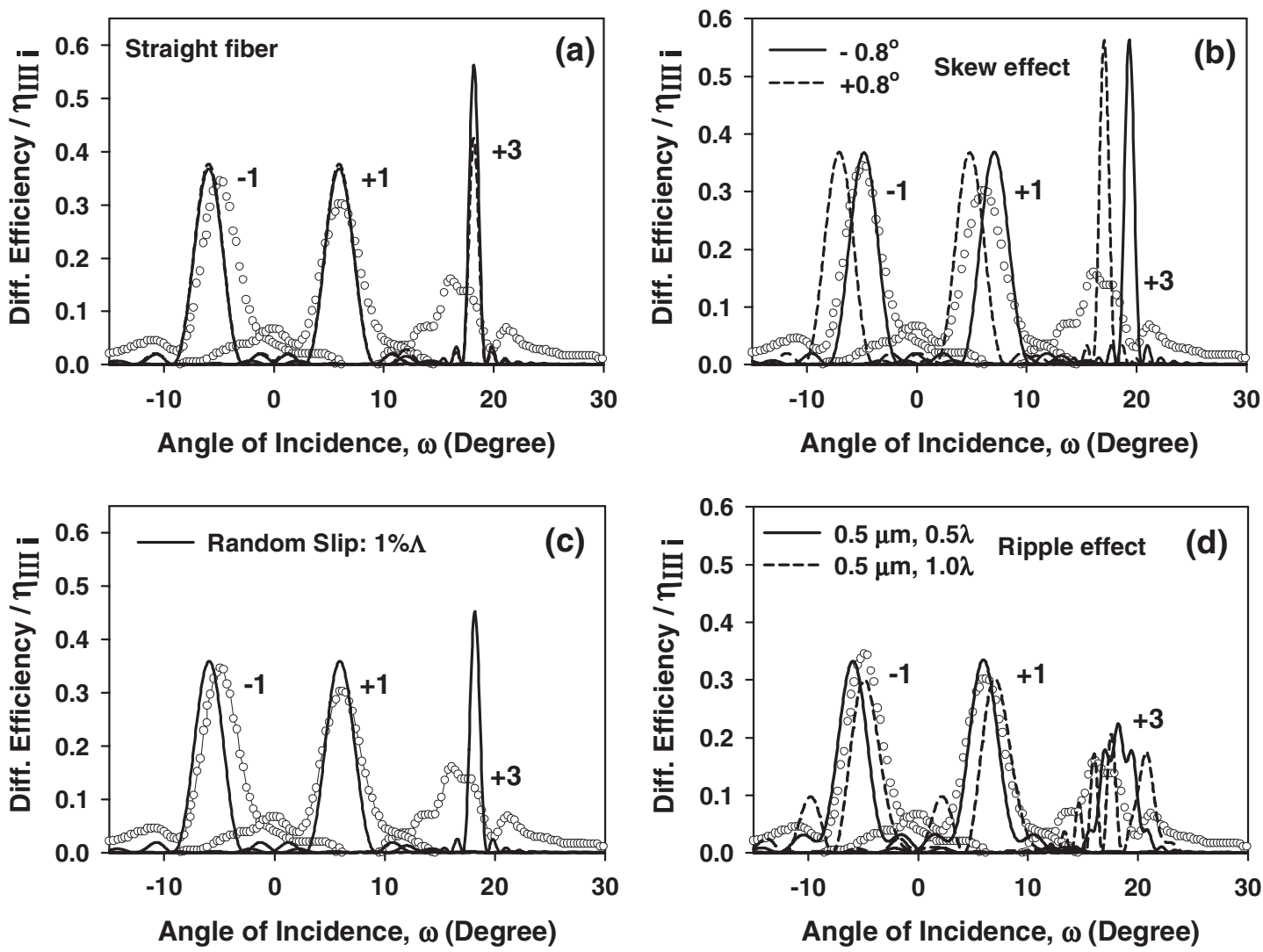

Fig. 4. Comparisons between experimental $\omega$ scans (curves of open circles) and theoretical results (solid curves) that were calculated for different myofibril parameters. Experimental results were obtained in rabbit pasos muscle fiber illuminated by TE polarized light. The sarcomere length was $\Lambda=3.04 \mu \mathrm{m}$ and the fiber thickness was $d=71 \mu \mathrm{m}$. Other parameters were as stated in Subsection 2.B. To conform to the experimental data, the refractive index outside the muscle fiber was 1.0. (a) Results obtained in straight fiber. (b) Effects of a positive (solid curve) or negative (dashed curve) skew $\left(\alpha_{\text {skew }}=0.8^{\circ}\right)$. (c) Effects of a random slip, $\delta_{\text {slip }}=1 \% \Lambda$. (d) Effects of half-wave ripple $\left(\lambda_{\text {ripple }}=2 d\right)$ and full-wave ripple $\left(\lambda_{\text {ripple }}=d\right)$ with ripple amplitude $u_{\text {ripple }}=0.5 \mu \mathrm{m}$.

ric grating [26]. The open circles in Fig. 4(a) show experimental results by Thornhill et al. [12] obtained using a focused TE polarized beam $(\sim 50 \mu \mathrm{m})$ in a rabbit $p a-$ sos muscle fiber. Diffraction peaks can be seen for three diffraction orders: $-1,+1$, and +3 . No second-order diffraction was observed at the specific sarcomere length $(3.04 \mu \mathrm{m})$ used. The Bragg condition predicts that the first-order peak will be at $5.98^{\circ}$ and the third-order peak will be at $18.2^{\circ}$. The measured angular difference between the +first-order and -first-order Bragg peaks was $10.96^{\circ}$, slightly less than the $11.96^{\circ}$ predicted from the Bragg angle condition.

The default sarcomere parameters discussed in subsection 2.B were applied. It can be seen that the calculated first- and second-order diffraction efficiencies agree closely with the experimental results. However, the thirdorder peak is both narrower and higher than that of the experimental results. We studied the effects of different sarcomere parameters and myofibril morphologies in our simulation. We found that variations in $W_{A}$ had minimal impact on the calculated diffraction efficiencies. For example, changing $W_{z}$ from 0.05 to 0.10 increased the diffraction efficiency by $6 \%$ in all diffraction orders. On the other hand, reducing $W_{A}$ from $1.5 \mu \mathrm{m}$ to $1.3 \mu \mathrm{m}$ increased first-order diffraction efficiency by only $2 \%$, but the third-order diffraction efficiency was reduced by $24 \%$.
We found that the refractive indices of individual sarcomere segments had more significant effects. An increase in the refractive index difference between the overlap A- and I-bands from $\Delta n=0.0074$ [12] to 0.0128 [31] caused a $124 \%$ increase in the first-order diffraction efficiency and a $77 \%$ increase in the third-order diffraction efficiency.

The skew effect shifts the $\omega$-scan results from those obtained in straight fibers [5,12,21,40]. As shown in Fig. 4(b), a skew angle of $0.8^{\circ}$ shifted the Bragg condition by $\sim 1.1^{\circ}$. To study the effect of random slip, we introduced a small slip that was equal to $1 \%$ of the sarcomere length $\delta_{\text {slip }}=1 \% \Lambda$, which was also used by Sidick et al. [16]. Figure 4(c) shows that there is essentially no change in the first-order diffraction, whereas the diffraction efficiency of the third order decreases by $19.6 \%$. Shifting effects were also observed when half-wave and full-wave ripples were applied [Fig. 4(d)]. In addition, a rippled muscle fiber changed diffraction efficiencies of all orders. In particular, the profiles of high diffraction orders were changed drastically. Following Thornhill et al. [12], we incorporated a small ripple of $0.5 \mu \mathrm{m}$ amplitude. As illustrated in Fig. 4(d), the small ripple significantly reduced the efficiency of the third-order diffraction and broadened its distribution.

The experimental data shown in Fig. 4 were obtained using an incident beam with a small illumination spot 
[12]. Within a small area, muscle fiber parameters are relatively uniform. Therefore multiple domain effects were unlikely to be significant [21]. When a larger incident beam with a $1 \mathrm{~mm}$ diameter was used, the $\omega$ scan showed obtained much wider distributions as observed by Rudel and Zite-Ferenczy [5] and Baskin et al. [40]. In Rudel and Zite-Ferenczy's study, a thick $(150 \mu \mathrm{m})$ frog (Rana esculenta) muscle fiber with a sarcomere length of $2.6 \mu \mathrm{m}$ was used (Fig. 5). The FWHMs of their $\omega$-scan peaks were more than $10^{\circ}$, much larger than the $<3^{\circ}$ data shown in Fig. 4. Due to domain effects, a different part of the illuminated fiber may have different myofibril profiles. Skew plays a dominant role in broadening the $\omega$-scan profile.
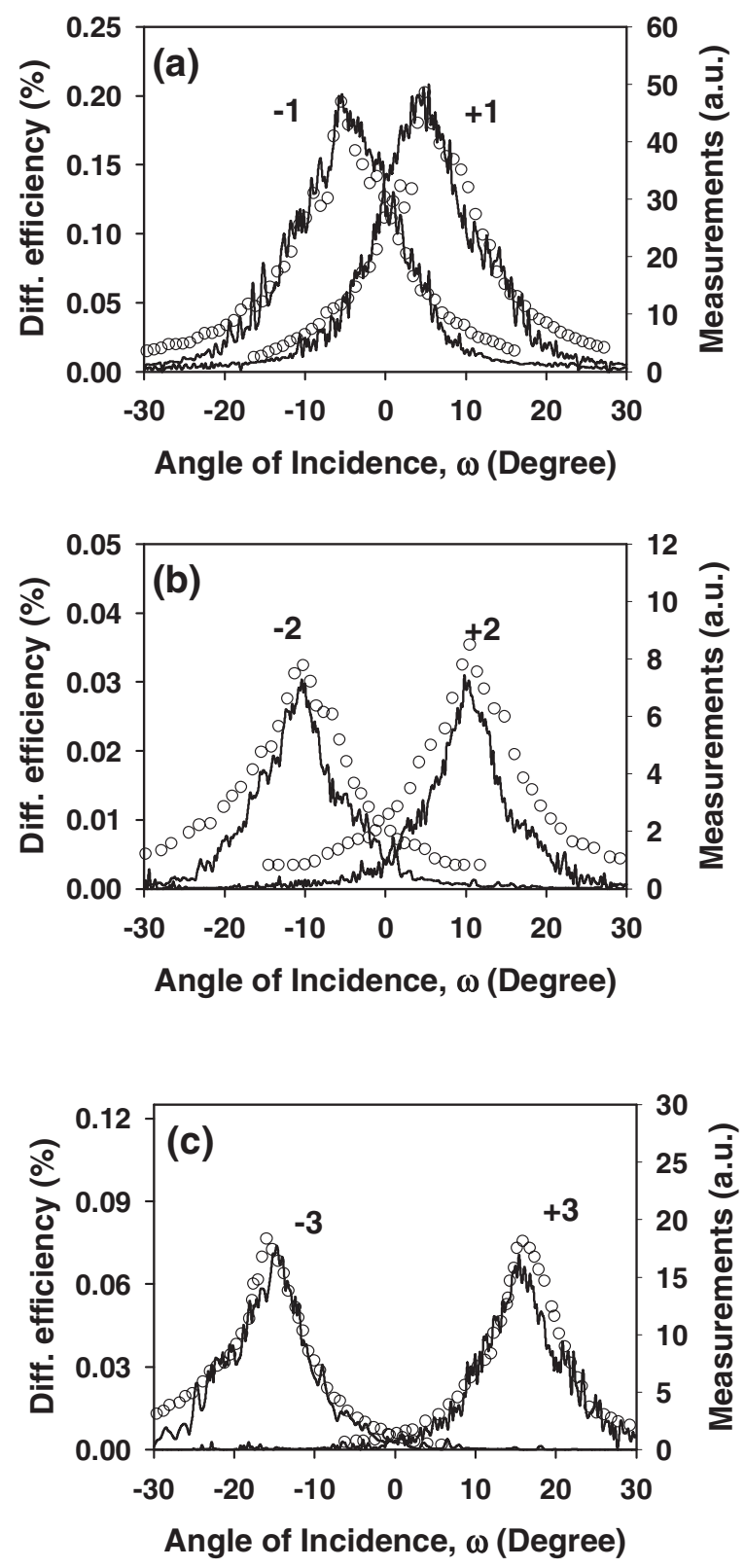

Fig. 5. Domain effect on $\omega$-scan profiles $(\Lambda=2.6 \mu \mathrm{m}, d$ $=150 \mu \mathrm{m})$. Open circles were defined according to experimental data from Rudel and Zite-Ferenczy [4]. The skew domain effect applied was $\alpha_{\nu}=5.9^{\circ}$ and $\alpha_{\mu}=0.0^{\circ}$. The default values discussed in Subsection 2.B were used for all other sarcomere parameters.
The summation of contributions from different domains significantly broadens the angular distribution because the skew effect shifts the $\omega$-scan profile.

In Fig. 5, we applied a domain effect using a Gaussian distributed skew angle [Eq. (12)] with a variance of $\alpha_{v}$ $=5.9^{\circ}$. The refractive index outside the muscle fiber was set to 1.377 (experimental mean refractive index of the fiber) in the calculation. To allow a quantitative comparison, the relative display scales among the first-, second-, and third-order diffractions were identical for both the simulation and the experiment. The simulated FWHMs were $13.2^{\circ}, 8.8^{\circ}$, and $9.7^{\circ}$ for the first-, second-, and thirdorder diffractions, respectively, while the corresponding experimental results were $12.7^{\circ}, 12.0^{\circ}$ and $8.7^{\circ}$. This result confirmed our hypothesis that the muscle domain effect can significantly broaden the $\omega$-scan profiles.

\section{B. Diffraction Efficiencies as a Function of Sarcomere Length}

Theoretical diffraction studies $[10,12,14,16]$ have suggested that oscillations exist at all diffraction orders when expressing diffraction efficiencies as a function of sarcomere length. This has been attributed to the effects of constructive and destructive interference. On the other hand, such effects have not been observed in experimental studies $[2,3]$. In addition, although it is known that the diffraction efficiency has a great dependence on sarcomere length [3], the experimental results obtained in frog semitendinosus muscles showed inconsistent trends. For example, in an early investigation, Buchthal and Knappeis [41] observed an increase in first-order diffraction with sarcomere length. Paolini et al. [2] reported that firstorder diffraction had a curvilinear relationship with sarcomere length and a peak diffraction that appeared at $\approx 2.8-3.0 \mu \mathrm{m}$. In contrast, Baskin et al. [3] reported that first-order diffraction increased with sarcomere length and reached a plateau at $\approx 3.8 \mu \mathrm{m}$. Kawai and Kuntz [9] also reported that first order diffraction increased with sarcomere length but at a much slower rate than Baskin's results [3] indicated. In [3], the signal increased 10 times when sarcomere length increased from $2.4 \mu \mathrm{m}$ to $4.0 \mu \mathrm{m}$, while [9] showed that the signal barely increased by a factor of 2 over the same range of sarcomere lengths.

We found that a variety of experiments under different test conditions yield results that can be explained by including a proper degree of skew and/or domain effects in the model. A summation of the diffractions from small segments with different skews eliminated the aforementioned oscillations as shown in Fig. 6, because a skew shifted the Bragg locations (Fig. 4). In addition, a different degree of domain effect altered the smoothing effect and therefore produced a different trend, as shown in Fig. 6.

Figure 6(a) shows that the curvilinear profile reported by Paolini et al. [2] can be obtained both in thin straight fibers and in skewed thicker fibers. The results of Baskin et al. [3] shown in Fig. 6(b) had a significantly different profile: the simulation outputs matched the experimental results in [3] very closely when a smaller domain effect of 

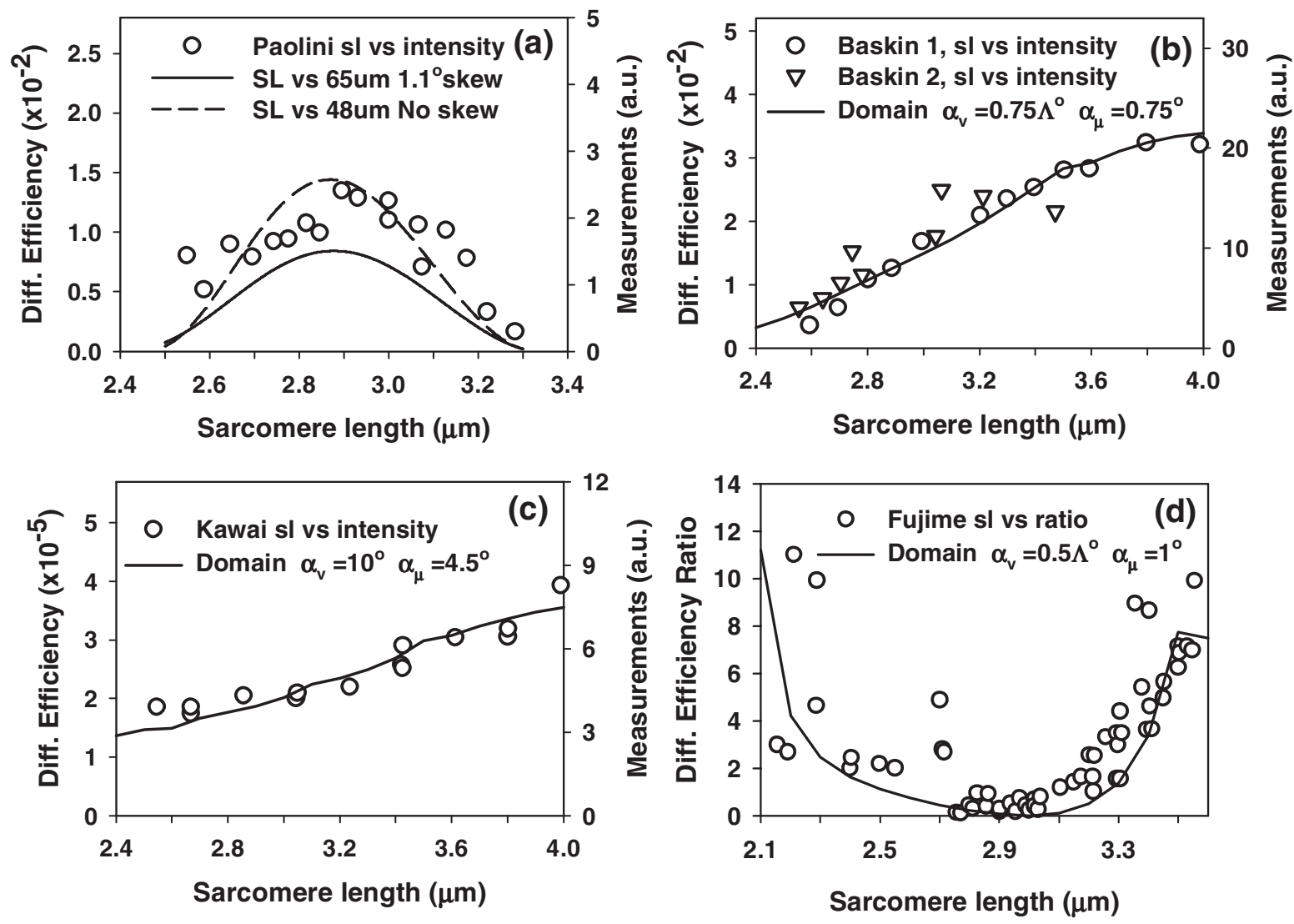

Fig. 6. Comparisons between simulated diffraction efficiencies (shown as solid curves) with experimental results (symbols) at different sarcomere lengths. (a) First-order diffraction measured by Paolini et al. [2] in single frog semitendinousus muscle fiber (circles). Also shown are the calculated results for a straight $48-\mu \mathrm{m}$-thick fiber (solid curve) and with $1.1^{\circ}$ skew for a 65 - $\mu$ m-thick fiber (dashed curve). (b) First-order diffraction measured by Baskin et al. [3] in single frog semitendinousus muscle fiber (circles and triangles). The simulation results were obtained by considering a domain effect $\left(\alpha_{\nu}=0.75 \times \Lambda^{\circ}\right.$ and $\left.\alpha_{\mu}=0.75^{\circ}\right)$. (c) First-order diffraction measured by Kawai and Kuntz [9] in single frog semitendinousus muscle fiber (circles). The simulation results were obtained by introducing a domain effect $\left(\alpha_{\nu}=10^{\circ}, \alpha_{\mu}=4.5^{\circ}\right)$. (d) Intensity ratio between the second and third diffraction orders measured by Fujime [7] in frog fascia of sartorius muscle fiber (circles). Theoretical values were calculated with a domain effect of skew using $\alpha_{\nu}=(0.5 \Lambda)^{\circ}$ and $\alpha_{\mu}=1.0^{\circ}$. TE polarization was used in all calculations. A common fiber thickness of $80 \mu \mathrm{m}$ was used for (b), (c), and (d). A value of $1.45 \mu \mathrm{m}$ was used as the A-band width for (d). The default values discussed in Subsection 2.B were used for all other sarcomere parameters.

skews was introduced. In the simulation, we assumed that the variance of the skew domain effect [Eq. (13)] increased linearly with sarcomere length: $\alpha_{v}=(0.75 \times \Lambda)^{\circ}$ with $\alpha_{\mu}=0.75^{\circ}$. Since fiber samples were stretched during experiments to achieve longer sarcomere lengths, it was likely that the inhomogeneity increased with sarcomere length.

In Kawai and Kuntz's experiment [9], a larger incident beam size $(2.0 \mathrm{~mm})$ was used, which potentially resulted in a more significant domain effect. Therefore we introduced a broader distribution of the skew domain effect $\left(\alpha_{v}=10^{\circ}, \alpha_{\mu}=4.5^{\circ}\right)$ in the simulation. As shown in Fig. 6(c), despite small discrepancies at shorter sarcomere lengths, the simulated trend was in good agreement with the experimental results.

Instead of providing absolute diffraction intensity, Fujime and Yoshino [6] calculated the intensity ratio between the second- and third-order diffractions at various sarcomere lengths. In straight fibers, this ratio has very large fluctuations due to the oscillations in each individual diffraction order. Thus the result cannot describe the observed experimental data shown in Fig. 6(d). However, by considering a smaller domain effect $\left[\alpha_{\mathrm{v}}=(0.5 \times \Lambda)^{\circ}, \alpha_{\mu}=1.0^{\circ}\right]$, the simulated results provided a good match with the experimental results.

\section{CONCLUSION}

We simulated laser diffraction in muscle fibers by applying the $3 \mathrm{DCW}$ algorithm to a theoretical sarcomere model. Actual muscle fibers are not perfectly uniform grating structures. Multiple irregularities may affect laser diffraction efficiency. By incorporating different irregularities in the model, a variety of experimental observations under different conditions can be explained.

The sarcomere parameters used in this study were all within normal physiological ranges as recorded in previous experiments. In some cases, the simulated results do not perfectly match experimental observations. This might be due to the fact that only the skew domain effect was considered in the simulation, while many other sarcomere parameters could have inhomogeneous distributions in the muscle fiber. In addition, the cylindrical nature of the muscle fibers is not considered in the model and its potential impact needs further study. 
Our results indicate that physiological inhomogeneities, as modeled with the domain effect, play a significant role in determining final diffraction efficiencies. With appropriate consideration of such inhomogeneous morphological properties in a physical sarcomere model, the 3DCW theory provides results that are consistent with experimental results of laser diffraction in single muscle fibers.

\section{ACKNOWLEDGMENTS}

This project was supported in part by National Science Foundation (NSF) grant CBET-0643190 and by the National Research Initiative (NRI) of the United States Department of Agriculture (USDA) Cooperative State Research, Education and Extension Service under grant 2006-35503-17619.

\section{REFERENCES}

1. B. R. Maclntosh, P. F. Gardiner, and A. J. McComas, Skeletal Muscle: Form and Function (Human Kinematics, 2006).

2. P. J. Paolini, R. Sabbadini, K. P. Roos, and R. J. Baskin, "Sarcomere length dispersion in single muscle fibers and fiber bundles," Biophys. J. 16, 919-930 (1976).

3. R. J. Baskin, K. P. Roos, and Y. Yeh, "Light diffraction study of single skeletal muscle fibers," Biophys. J. 28, 45-64 (1979).

4. R. Rudel and F. Zite-Ferenczy, "Efficiency of light diffraction by cross striated muscle fibers under stretch and during isometric contraction," Biophys. J. 30, 507-516 (1980).

5. R. Rudel and F. Zite-Ferenczy, "Interpretation of light diffraction by cross-striated muscle as Bragg reflexion of light by the lattice of contractile proteins," J. Physiol. (London) 290, 317-330 (1979).

6. S. Fujime and S. Yoshino, "Optical diffraction study of muscle fibers. I. A theoretical basis," Biophys. Chem. 8, 305-315 (1978).

7. S. Fujime, "Optical diffraction study of muscle fibers," Biochim. Biophys. Acta 37, 227-238 (1975).

8. Y. Yeh, R. J. Baskin, R. L. Leiber, and K. P. Roos, "Theory of light diffraction by single skeletal muscle fibers," Biophys. J. 29, 509-522 (1980).

9. M. Kawai and I. D. Kuntz, "Optical diffraction studies of muscle fibers," Biophys. J. 13, 857-876 (1973).

10. A. F. Huxley, "Theoretical treatment of diffraction light by a striated muscle fibre," Proc. R. Soc. London, Ser. B 241, 65-71 (1990).

11. H. E. Huxley and J. Hanson, "Quantitative studies on the structure of cross striated myofibrils.," Biochim. Biophys. Acta 23, 229-249 (1957).

12. R. A. Thornhill, N. Thomas, and N. Berovic, "Optical diffraction by well ordered muscle fibers," Eur. Biophys. J. 20, 87-99 (1991).

13. H. Kogelnik, "Coupled wave theory for thick hologram grating,” Bell Syst. Tech. J. 48, 2909-2943 (1969).

14. E. Sidick, J. Knoesen, K. Xian, Y. Yeh, and R. J. Baskin, "Rigorous analysis of light diffraction by a striated muscle fibre," Proc. R. Soc. London, Ser. B 249, 247-256 (1992).

15. M. G. Moharam and T. K. Gaylord, "Rigorous coupled wave analysis of planar grating diffraction,” J. Opt. Soc. Am. 71, 811-818 (1981)

16. E. Sidick, R. J. Baskin, Y. Yeh, and J. Knoesen, "Rigorous analysis of light diffraction ellipsometry by striated muscle fibers," Biophys. J. 66, 2051-2061 (1994).

17. A. F. Leung, "Calculation of the laser diffraction intensity of striated muscle by numerical methods," Comput. Programs Biomed. 15, 169-174 (1982).

18. M. M. Judy, T. Summerour, R. Leconey, R. L. Roa, and G. H. Templeton, "Muscle diffraction theory," Biophys. J. 37, 475-487 (1982).
19. S. Ishiwata, K. Muramatsu, and H. Higuchi, "Disassembly from both ends of thick filaments in rabbit skeletal muscle fibers," Biophys. J. 47, 257-266 (1985).

20. R. L. Moss, "Sarcomere length-tension relations of frog skinned muscle fibers during calcium activation at short length,” J. Physiol. (London) 292, 177-192 (1979).

21. B. Brenner, "Sarcomeric domain organization within single skinned rabbit psoas fibers and its effects on laser light diffraction patterns," Biophys. J. 48, 967-982 (1985).

22. C. L. Sundell, Y. E. Goldman, and L. D. Peachey, "Fine structure in near-field and far-field laser diffraction patterns from skeletal muscle fibers," Biophys. J. 49, 521-530 (1986).

23. R. L. Leiber, Y. Yeh, and R. J. Baskin, "Sarcomere length determination using laser diffraction," Biophys. J. 45 1007-1016 (1984).

24. M. G. Moharam and T. K. Gaylord, "Three-dimensional vector coupled-wave analysis of planar grating diffraction," J. Opt. Soc. Am. 73, 1105-1112 (1983).

25. J. Ranasinghesagara and G. Yao, "Imaging 2D optical diffuse reflectance in skeletal muscle," Opt. Express 15, 3998-4007 (2007).

26. T. K. Gaylord and M. G. Moharam, "Analysis and application of optical diffraction by grating," Proc. IEEE 73, 894-937 (1995).

27. N. P. van der Aa and R. M. M. Mattheij, "Computing shape parameter sensitivity of the field of one-dimensional surface-relief gratings by using an analytical approach based on RCWA,” J. Opt. Soc. Am. A 24, 2692-2700 (2007).

28. M. G. Moharam, A. Drew, A. Pommet, E. B. Grann, and T. K. Gaylord, "Stable implementation of the rigorous coupled-wave analysis for surface-relief gratings: enhanced transmittance matrix approach,” J. Opt. Soc. Am. A 12, 1077-1086 (1995).

29. M. G. Moharam, E. B. Grann, A. Pommet, and T. K. Gaylord, "Formulation for stable and efficient implementation of the rigorous coupled-wave analysis of binary gratings,” J. Opt. Soc. Am. A 12, 1068-1076 (1995).

30. P. Lalanne, "Improved formulation of the coupled-wave method for two-dimensional gratings," J. Opt. Soc. Am. A 14, 1592-1598 (1997).

31. A. F. Huxley and R. Niedergerke, "Measurement of the striations of isolated muscle fibers with the interference microscope," J. Physiol. (London) 144, 403-425 (1958).

32. S. G. Page and H. E. Huxley, "Filament lengths in striated muscle," J. Cell Biol. 19, 369-390 (1963).

33. F. Carlsen, G. G. Knappeis, and F. Buchthal, "Ultrastructure of the resting and contracted striated muscle fiber at different degrees of stretch," J. Biophys. Biochem. Cytol. 11, 95-117 (1961).

34. W. Ding, H. Fujita, and M. Kawai, "The length of cooperative units on the filament in rabbit psoas muscle fibres," Exp. Physiol. 87, 691-697 (2002).

35. P. K. Luther, R. Padron, S. Ritter, R. Craig, and J. M. Suire, "Hetrogenity of Z-band structure within a single muscle sarcomere: implications for sarcomere assembly," J. Mol. Biol. 332, 161-169 (2003).

36. G. G. Knappeis and F. Carlsen, "The ultrastructure of the Z disc in skeletal muscle," J. Cell Biol. 13, 323-335 (1962).

37. A. F. Leung, Y. M. Cheung, and J. C. Hwang, "Light diffraction intensity from muscle fibers in different osmotic solutions: measurement of equilibrium time," Eur. J. Physiol. 414, 676-682 (1989).

38. K. Burton and A. F. Huxley, "Identification of source of oscilations in apparent sarcomere length measured by laser diffraction," Biophys. J. 68, 2429-2443 (1995).

39. Y. Yeh and R. J. Baskin, "Thory of optical ellipsometric measurements from muscle diffraction studies," Biophys. J. 54, 205-218 (1988).

40. R. J. Baskin, R. L. Leiber, T. Oba, and Y. Yeh, "Intensity of light diffraction from striated muscle as a function of incident angle," Biophys. J. 36, 759-773 (1981).

41. F. Buchthal and G. G. Knappeis, "Diffraction spectra and minute structure of the cross-striated muscle fiber," Skand. Arch. Physiol. 83, 281-307 (1940). 